\title{
Flotillin-1 facilitates toll-like receptor 3 signaling in human endothelial cells
}

\author{
Christian Fork · Juliane Hitzel · Benjamin J. Nichols • \\ Ritva Tikkanen $\cdot$ Ralf P. Brandes
}

Received: 6 May 2014/Revised: 22 August 2014/Accepted: 3 September 2014/Published online: 10 September 2014

(C) Springer-Verlag Berlin Heidelberg 2014

\begin{abstract}
Endothelial cells are important elements in the vascular response to danger-associated molecules signaling through toll-like receptors (TLRs). Flotillin-1 and -2 are markers of membrane rafts but their true endothelial function is unknown. We hypothesized that flotillins are required for TLR signaling in human umbilical vein endothelial cells (HUVECs). Knockdown of flotillin-1 by shRNA decreased the TLR3-mediated poly-I:C-induced but not the TLR4mediated LPS-induced inflammatory activation of HUVEC. As TLR3 but not TLR4 signals through the endosomal compartment, flotillin-1 might be involved in the transport of poly-I:C to its receptor. Consistently, uptake of poly-I:C was attenuated by flotillin-1 knockdown and probably involved the scavenger receptor SCARA4 as revealed by knockdown of this receptor. To determine the underlying mechanism, SILAC proteomics was performed. Down-
\end{abstract}

Electronic supplementary material The online version of this article (doi:10.1007/s00395-014-0439-4) contains supplementary material, which is available to authorized users.

C. Fork · J. Hitzel · R. P. Brandes $(\bowtie)$

Institute for Cardiovascular Physiology, Medical Faculty,

Goethe-University Frankfurt, Theodor Stern Kai 7,

60690 Frankfurt, Germany

e-mail: brandes@vrc.uni-frankfurt.de

C. Fork $\cdot$ R. P. Brandes

German Center for Cardiovascular Research (DZHK),

Partner site RheinMain, Frankfurt, Germany

B. J. Nichols

MRC Laboratory of Molecular Biology, Cambridge CB2 0QH,

UK

R. Tikkanen

Institute of Biochemistry, Medical Faculty, University of

Giessen, Gießen, Germany regulation of flotillin-1 led to a reduction of the structural caveolae proteins caveolin-1, cavin-1 and -2 , suggesting a role of flotillin-1 in caveolae formation. Flotillin-1 and caveolin-1 colocalized within the cell, and knockdown of flotillin-1 decreased caveolin-1 expression in an endoplasmic reticulum stress-dependent manner. Importantly, downregulation of caveolin-1 also attenuated TLR3-induced signaling. To demonstrate the importance of this finding, cell adhesion was studied. Flotillin-1 shRNA attenuated the poly-I:C-mediated induction of the adhesion molecules VCAM-1 and ICAM-1. As a consequence, the poly-I:Cinduced adhesion of peripheral blood mononuclear cells onto HUVECs was significantly attenuated by flotillin-1 shRNA. Collectively, these data suggest that interaction between flotillin-1 and caveolin-1 may facilitate the transport of TLR3-ligands to its intracellular receptor and enables inflammatory TLR3 signaling.

Keywords Endothelium - Lipid rafts · Caveolae - Cavin . Inflammation - Toll-like receptors - Scavenger receptors

\section{Introduction}

Endothelial cells are the gate keepers of vascular inflammatory signaling. In acute inflammation, the endothelium attracts and guides leukocytes to the site of affliction. Chronic endothelial activation promotes atherosclerosis development and aneurysm formation. Endothelial cells sense danger-associated molecules (DAMPs) by pattern recognition receptors, like toll-like receptors (TLR1-9) of the innate immune system. On the long run, this may promotes vascular disease as cholesterol and other unspecific pro-atherosclerotic agents are also sensed by TLRs [31]. In the acute setting of inflammation, however, TLR 
signaling is needed to facilitate the local endothelial response to pathogens.

TLR signaling is complex and of the several elements involved, the MyD88- and TRIF-pathways are of particular relevance. MyD88 activates nuclear factor (NF)- $\mathrm{kB}$, which induces inflammatory cytokine expression. The TRIF pathway stimulates interferon regulatory factor-3 (IRF3), in addition to NF- $\mathrm{KB}$ and induces type I interferons [23]. Importantly, TLR3 does not really activate MyD88 and therefore only stimulates the TRIF pathway [27].

On the basis of their subcellular localization, TLRs are divided in two groups. TLR1, 2, 4, 5 and 6 are expressed on the cell surface where they recognize lipid structures and, in the case of TLR5, the protein flagellin. TLR3, 7, 8 and 9 reside intracellularly and sense nucleic acids within the endolysosomal compartment [28]. Their intracellular localization is potentially a consequence of the fact that TLR binding to nucleic acids requires an acidic environment [20]. Thus, circulating nucleic acids have to be delivered to intracellular TLRs. The mechanisms underlying this transport are, however, not completely characterized but obviously have to involve endocytosis of the nucleic acid. At least two major endocytotic pathways operate in parallel in endothelial cells: uptake through clathrin-coated pits and internalization by membrane rafts [10]. Although the concept of membrane rafts is still vigorously debated, there is consensus that proteins of the flotillin family are enriched in this fraction and are markers of rafts [32].

Interestingly, despite their ubiquitous expression and high conservation, the physiological function of flotillins is far from clear and their role in the endothelium has, to our knowledge, not yet been determined. The flotillin protein family consists of flotillin-1 and 2 (also called reggies) which homo- and hetero-oligomerize [2, 37]. Despite some other functions [4, 32], flotillins have been linked to endocytosis and vesicular trafficking but also to signaling $[6,8,14,15,24,33,36,38]$. For example, flotillins modulate epidermal growth factor receptor auto-phosphorylation and activation of downstream target molecules [1].

Considering their prominent positioning on lipid rafts, we here hypothesize that flotillins contribute to vascular inflammatory signaling in response to the endosomal TLR3 and studied this in human umbilical vein endothelial cells (HUVECs).

\section{Materials and methods}

Materials

Human fibronectin was purchased from BD Biosciences (Heidelberg, Germany). DAPI, chloroquine, carrageenan, dextran sulfate, lipopolysaccharide (E. coli), filipin III and methyl- $\beta$-cyclodextrin from Sigma-Aldrich. MG132 was from Merck, Leupeptin and pepstatin from Applichem. Poly-I:C high molecular weight, Poly-I:C-rhodamine and lipoteichoic acid (S. aureus) were from InvivoGen (San Diego, USA). Phenylmethylsulfonyl fluoride (PMSF) was from Applichem.

\section{Cell culture}

Four batches of HUVECs were purchased from Lonza (Walkersville, MD). Cells were cultured in EBM (Lonza, Walkersville, MD), supplemented with $8 \%$ FCS, bovine brain supplement and human recombinant EGF, penicillin $(50 \mathrm{U} / \mathrm{ml})$ and streptomycin $(50 \mu \mathrm{g} / \mathrm{ml})$. Cells of passage two to four were used throughout the study and all experiments were performed at least in three different batches. Cells were stimulated in EBM with $1 \%$ FCS, except thapsigargin treatment it was performed in EBM with $4 \%$ FCS. Concentration and timing of the experiments are described in the figure legend. For poly-I:C-rhodamine uptake, cells were stimulated for $3 \mathrm{~h}(0.5 \mu \mathrm{g} / \mathrm{ml})$ washed with phosphate buffered saline and subsequently images were acquired by confocal microscopy (LSM 510, Zeiss).

siRNA, shRNA and plasmid transfection

For shRNA treatment, endothelial cells were infected with lentiviral particles according to Addgene "plKO.1 Protocol" (http://www.addgene.org/tools/protocols/plko/). Cells were selected with puromycin $(0.5 \mu \mathrm{g} / \mathrm{ml})$. The shFlot-1A target sequence was: 5'-CAGAGAAGUCCCAACUAA UUA-3' ${ }^{\prime}$, for shFlot-1B 5'-CUGCUUGGCUUUAGCUUCC CG- $3^{\prime}$ and for m-shFlot-1 CAGGATTACTTACACTCGT TA. Unless otherwise noted, all experiments were performed with shFlot-1A. Target sequence for shFlot-2 was 5'-GUGCAGAGAGAUGCUGACAUU-3'. Control shRNA against green fluorescent protein (shGFP) and shScrambled (shScr) were from Addgene. For siRNA treatment, endothelial cells (80-90\% confluent) were transfected with GeneTrans II according to the instructions provided by MoBiTec (Göttingen, Germany). All siRNAs (Stealth RNAi) were from Invitrogen. siScrambled (siScr) was used as a negative control. Plasmid overexpression was achieved with the neon electroporation system (Invitrogen). The plasmids pFlot-1-GFP-N1 and pFlot-2-GFP-N1 have been described earlier [30]. Control plasmid mCherry-N1 was from Clontech. Two siRNAs and shRNAs were used to exclude that unspecific effects of an individual approach mediated the effects observed.

\section{Quantitative RT-PCR}

Total RNA was extracted with the RNA Mini Kit (Bio\&Sell). cDNA was prepared with SuperScript III reverse 
transcriptase (Invitrogen) and random hexamer primers. Quantitative real-time PCR was performed with Fast Plus Eva Green Master Mix and ROX as reference dye (Biotium) in an Mx3005 cycler (Stratagene) with primer sequences as follows: VCAM-1 forward primer, $5^{\prime}$ TGTCATCATCTCTTGTACATGTGG- $3^{\prime}$, and reverse primer, 5'-GTTTTCTCTTCCTTGAACATCAAG-3'; ICA M-1 forward primer, 5'-CAGTGGGCAA-GAACCTTAC CCTAC- $3^{\prime}$, and reverse primer, 5'-GTTCAGTGCGG CACGAGAAATTGG-3'; IFNB forward primer, 5'- AG TGTCAGAAGCTCCTGTGGC- $3^{\prime}$ and reverse $5^{\prime}$ - TGAGG CAGT ATTCAAGCCTCC-3'; CXCL10 forward primer, $5^{\prime}$-TGGCATTCAAGGAGTACCTCTCT- ${ }^{\prime}$ and reverse $5^{\prime}$ CTGATGCAGGTACAGCGTACG-3', SCARA1 forward primer, $5^{\prime}$ - CTC CAT TTA CGA AAG TTC GAC TG- ${ }^{\prime}$ and reverse $5^{\prime}$-ACCAGTACCTTGTCCAAAGTG- $3^{\prime}$; SC ARA2 forward primer, 5'-AAATCAATGTTCCAAAGCC CAAG- $3^{\prime}$ and reverse $5^{\prime}$-ACTGCAGCAAGG AGAAG GAC-3'; SCARA3 forward primer, 5'-TGGATCCGAAA GCCCTGAAC- $3^{\prime}$ and reverse $5^{\prime}$-AGAAGGAGCAACTG CCCATC-3'; SCARA4 forward primer, 5'-GAGCGTGAA AATGAA TGGAAGTG- $3^{\prime}$ and reverse $5^{\prime}$-AATGCAG ATGACAGTACTGTCTC-3'; SCARA5 forward primer, $5^{\prime}$-TCTTCATCTTAGCAGTGTCCAG-3' and reverse $5^{\prime}$ GCAACGAGTCTGACTGGT TC- ${ }^{\prime}$; SCARB1 forward primer, $5^{\prime}$-CAGCAGGTCCTTAAGAACGTG- $3^{\prime}$ and reverse $5^{\prime}$-GTT GTTGTTGAAGGTGATGTTGC-3'; SCA RB3 forward primer, 5'-CTATGCTGTATTTGAATC CGACG- $3^{\prime}$ and reverse $5^{\prime}$-TACACAGGTCTCCCTTCT TTG-3'; SCARF1 forward primer, 5'-AGGATGAAGCTG CAGGTCTG- $3^{\prime}$ and reverse $5^{\prime}$-ACAGGGACCATCCCT TCTTG- $3^{\prime}$. Caveolin-1 forward primer-A, 5'-ACGTGGT CAAGATTGACTTTGAAG- $3^{\prime}$ and reverse-A 5'-AGA TGTGCAGGAAAGAGAGAATG- $3^{\prime}$. Caveolin-1 forward primer-B, 5'-TAGACTCGGAGG GACATCTC- $3^{\prime}$ and reverse-B 5'-TCGATCTCCTTGGTGTGCGCGTC- ${ }^{\prime}$. Caveolin-2 forward primer, 5'-TCGCATCTCAAGCTG GGCTTCG-3' and reverse 5'-TACAAAAGG CATT AAAATCCAGATG-3' ${ }^{\prime}$. Flotillin-1 forward primer, 5' AAGCTGCCCCAGGTGGCAGAG G-3' and reverse $5^{\prime}$ TGTTCTCAAAGGCTTGTGATTCACC- $3^{\prime}$. Flotillin-2 forward primer, 5'-AGGGTGAAAAGGTGAAGCAGGT CC- $3^{\prime}$ and reverse $5^{\prime}$-TTGGCAGCA ATCTGGGGCAG G-3'. Relative mRNA level of target genes was normalized to polymerase (RNA)II (DNA-directed) polypeptide A (POLR2A) with the primer pair 5'-GCACCACGTCCAA TGACAT- $3^{\prime}$ and $5^{\prime}$-GTGCGGCTGCTTCCATAA- ${ }^{\prime}$, analyzed by the $\Delta \Delta \mathrm{CT}$ method.

Protein isolation and Western blot analysis

Cells were lysed in buffer containing $10 \mathrm{mmol} / \mathrm{l} \mathrm{Tris} / \mathrm{HCl}$, $\mathrm{pH} 8.0,150 \mathrm{mmol} / \mathrm{l} \mathrm{NaCl}, 10 \mathrm{mmol} / \mathrm{l}, 5 \mathrm{mmol} / \mathrm{l}$ EDTA,
$0.5 \%$ Triton X-100, $60 \mathrm{mmol} / \mathrm{l} \mathrm{N}$-octylglucoside, supplemented with $40 \mathrm{mg} / \mathrm{l}$ PMSF and protease inhibitor mix (antipain, aprotinin, leupeptin, chymostatin, pepstatin, trypsin inhibitor; $2 \mathrm{mg} / \mathrm{ml}$ each; all from AppliChem). After determination of protein concentration by Bradford assay, equal amounts of proteins were boiled in Laemmli buffer and separated by SDS-PAGE gel. After transfer of proteins onto nitrocellulose membranes and blocking with Roti-Block (Carl Roth), the following primary antibodies were used: anti-flotillin-1, flotillin-2 both from BD Biosciences $(610821,610384)$ and Santa Cruz (sc-25506, sc25507). Caveolin-1 and caveolin-2 from Abnova (MAB2408) and Cell Signaling (8522). The antibody for VCAM-1(sc-8304), ICAM-1(sc-8439) and p65(sc-109) were from Santa Cruz, GAPDH (G8795) from SigmaAldrich. IRF-3 (4302) and pIRF-3 (Ser 385) (4947) were from Cell Signaling. PERK (Thr 981) (sc-32577) and eIF2 $\alpha$ (Ser52) (sc-101670) from Santa Cruz. Western blot analysis was performed with an infrared-based laser scanning detection system (Odyssey, Licor). The infrared fluorescent-dye-conjugated secondary antibodies were purchased from Licor.

\section{SILAC LTQ-Orbitrap mass spectrometry} and detergent-resistant membrane isolation

Stable isotope labeling of amino acids (SILAC) was performed as described in the manufacturer's protocol from Thermo Scientific. The "SILAC protein quantitation kitDMEM:F12" was used. Simultaneously to shRNA transduction, knockdown cells were incubated with "heavy" ${ }^{13} \mathrm{C}_{6}$ L-lysine $+{ }^{13} \mathrm{C}_{6}^{15} \mathrm{~N}_{4}$ L-arginin and control shRNA cells with "light" ${ }^{12} \mathrm{C}_{6}$ L-lysine $+{ }^{12} \mathrm{C}_{6}^{14} \mathrm{~N}_{4}$ L-arginin for 9 days. Cell medium was supplemented with dialyzed $10 \%$ FBS, $0.4 \%$ ECGS $/ \mathrm{H}, 10 \mathrm{ng} / \mathrm{ml}$ epidermal growth factor receptor, $1 \mu \mathrm{g} / \mathrm{ml}$ hydrocortisone and proline $500 \mu \mathrm{mol} / \mathrm{l}$. Heavy and light labeled cell lysates were pooled according to their total protein concentration. Detergent-resistant membranes were isolated as reported previously by others [17] employing a three-step sucrose gradient of 40, 30 and $5 \%$. Detergent-resistant membranes accumulated according to their density at the interface between the 30 and $5 \%$ sucrose layer after ultracentrifugation. Samples were digested with trypsin $(15 \mathrm{~h})$ followed by dithiothreitol (DTT) and iodoacetamide treatment and purified by solid-phase extraction (SPE), subsequently subjected to Orbitrap XL mass spectrometer (Thermo) coupled to a nano-HPLC (Agilent). Chromatography was performed on a C18-reversed phase silica filled column in a $75 \mu \mathrm{m}$ ID PicoTip emitter (New Objectives) in $60 \mathrm{~min}$ HPLC runs using gradients with $0.1 \%$ formic acid from 5 to $50 \%$ acetonitrile following by $90 \%$ wash and $5 \%$ reequilibration steps. Eluted peptides were analyzed by MS/ 
MS method in positive mode programmed to fragment the top ten most abundant ions using dynamic exclusion with a resolution of 30,000 at $400 \mathrm{Th}$. Single charged precursor ions were rejected, the fragmentation of peptide ions occurred in the linear ion trap by CID at $35 \%$ collision energy. Fragmentation spectra were extracted from RAW spectra using extract_msn (Thermo) and matched against the human database (Uniprot) containing 20,366 sequences with the Mascot server 2.2 search engine. The search parameters were set as following: $15 \mathrm{ppm}$ deviation on the precursor and 0.6 Da on fragment masses, fixed carbamidomethylation of cysteine, variable oxidation of methionine and one missed cleavage. Only peptides with Mascot scores above the significance threshold of 0.05 were taken into account.

Fluorescence-activated cell sorting (FACS)

For FACS analysis, cells were detached with Versene and incubated with TLR3 or TLR4 antibodies (Abcam) for $45 \mathrm{~min}$ by $4{ }^{\circ} \mathrm{C}$. After washing, cells were incubated with the secondary antibody conjugated with Alexa Fluor 488 (45 min, $4{ }^{\circ} \mathrm{C}$ ). For intracellular staining, cells were permeabilized with Cytofix/Cytoperm (BD Biosience) for $20 \mathrm{~min}$ by $4^{\circ} \mathrm{C}$, before the primary antibody was added. Cells were analyzed by the FACS calibur machine (BD Biosciences, Heidelberg, Germany).

Proximity ligation assay (PLA) and total internal reflection fluorescence (TIRF) microscopy

PLA was performed with the Duolink kit as described in the manufacturer's protocol (Duolink II Fluorescence, OLink, Uppsala, Sweden). HUVECs were fixed in phosphate buffered formaldehyde solution (4\%), permeabilized with Triton X-100 (0.2\%), blocked with serum albumin solution (3\%) in phosphate buffered saline and incubated over night with antibodies against caveolin-1 (Abnova) and flotillin-1 (Cell Signaling). After washing, samples were incubated with the respective PLA-probes for $1 \mathrm{~h}\left(37^{\circ} \mathrm{C}\right)$, washed and ligated for $30 \mathrm{~min}\left(37^{\circ} \mathrm{C}\right)$. After an additional washing, amplification with polymerase was allowed for $100 \mathrm{~min}\left(37^{\circ} \mathrm{C}\right)$. The basal plasma membrane was shown by paxillin (BD Bioscience) and the nuclei were stained using DAPI. Images were acquired by Zeiss TIRF System LASOS77 in epifluorescence and TIRF mode, which only illuminates the first $200 \mathrm{~nm}$ of the basal compartment of the cell.

Peripheral blood mononuclear cell (PBMC) adhesion

$2 \times 10^{4}$ HUVECS were seeded on $\mu$-slides (IBIDI VI 0.4) and cultured to $95 \%$ confluence, subsequently stimulated with poly-I:C $(10 \mu \mathrm{g} / \mathrm{ml}, 5 \mathrm{~h})$ and incubated with PBMC (provided by DRK-Blutspendedienst, Germany, Hessen) for $3 \mathrm{~min}$. To distinguish between attached and nonattached PBMCs, the chamber was subsequently superfused with Hank's buffer containing $0.5 \%$ bovine serum albumin. Starting with an initial flow rate of $0.35 \mathrm{dyn} / \mathrm{cm}^{2}$, perfusion was increased to $2,5,8$ and $15 \mathrm{dyn} / \mathrm{cm}^{2}$ every 30 s. Adherent PBMCs were quantified by microscopy (AxioObserver, Zeiss).

\section{Statistics}

Data are expressed as the mean \pm SEM. Statistical analyses were performed by students $t$ test or one-way ANOVA followed by Bonferronís multiple comparison test for normally distributed values according to $F$ test. For notnormally distributed values, Kruskal-Wallis-Test followed by Dunns correction for the comparison of more than two groups or Mann-Whitney-Test for two independent groups was applied. For paired comparisons of two dependent groups the Mann-Whitney-Wilcoxon-Test was used. Values of $P<0.05$ were considered statistically significant.

\section{Results}

Flotillin-1 is essential for poly-I:C-induced inflammatory gene expression

The role of flotillin-1 in TLR-induced signaling in human umbilical vein endothelial cells (HUVEC) was studied by shRNA and TLR signaling was initiated by selective agonists. Activation of TLR3 by polyriboinosinic polyribocytidylic acid (poly-I:C) and of TLR4 by lipopolysaccharide (LPS) induced endothelial inflammatory adhesion molecule expression, whereas the TLR2 ligand lipoteichoic acid had little effect in endothelial cells (Fig. 1a, b). Knockdown of flotillin-1 decreased the poly-I:C-induced VCAM-1 and ICAM-1 expression (Fig. 1a, c), whereas flotillin-1 shRNA did not affect LPS-induced inflammatory activation (Fig. 1b, c). Basal level of VCAM-1 and ICAM-1 was not affected by the control or flotillin-1 shRNA (data not shown).

Flotillin-1 is involved in poly-I:C-induced IRF-3 signaling

Endothelial poly-I:C-induced signaling activates the TRAF-MyD88 pathway as well as the transcription factor IRF-3, which facilitates type I interferon expression [27]. Down-regulation of flotillin-1 also attenuated the poly-I:Cinduced expression of interferon $\beta$ (IFN $\beta$ ) as well as of the interferon related cytokine CXCL10 (Fig. 2a). As 


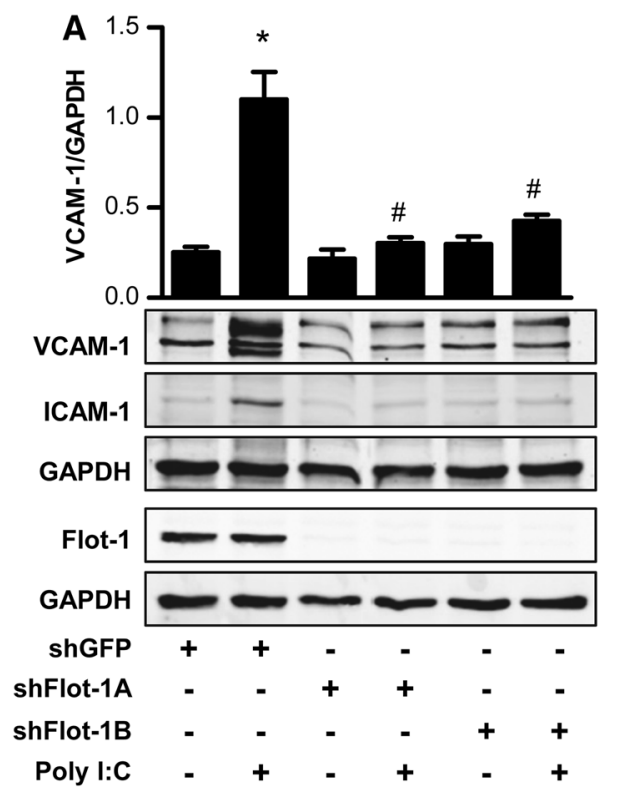

Fig. 1 Flotillin-1 is essential for poly-I:C-induced inflammatory gene expression. a and $\mathbf{b}$ Western blot and densitometry for the proteins indicated in HUVEC treated with or without poly-I:C, lipopolysaccharide (LPS) and lipoteichonic acid (LTA, each $10 \mu \mathrm{g} / \mathrm{ml}, 3 \mathrm{~h}$ ) after lentiviral transduction with shRNAs for flotillin-1 (shFlot-1A and $B$ ) or GFP (shGFP) (c) qRT-PCR for VCAM-1 relative to RNA
B

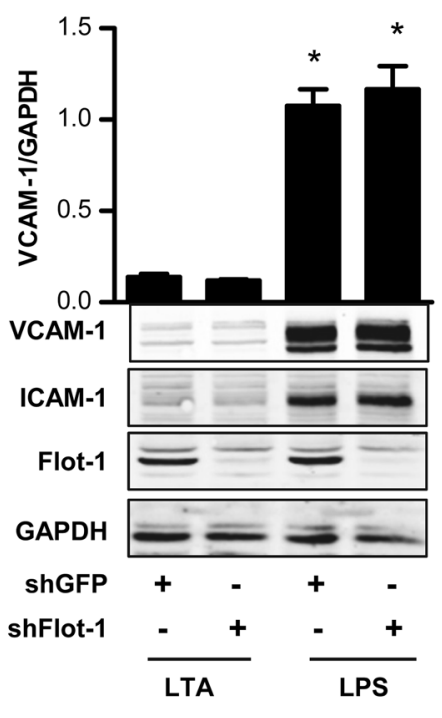

C

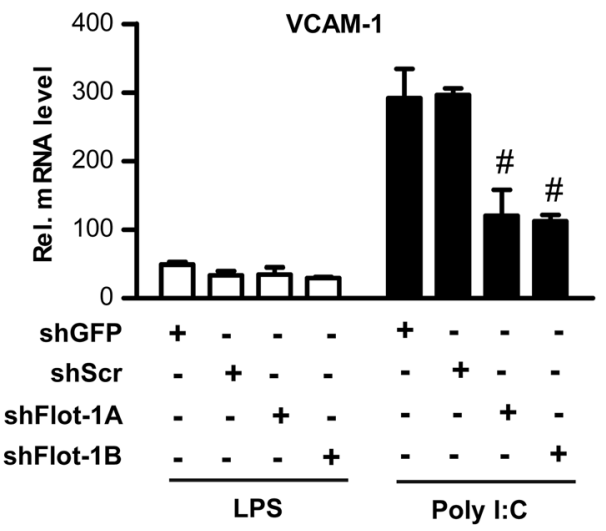

expected, TLR4 activation, which predominantly signals through MyD88 only induced a weak activation of the IRF pathway and importantly, this response was not affected by down-regulation of flotillin-1. In contrast, the poly-I:Cinduced phosphorylation of IRF-3 was attenuated by downregulation of flotillin-1 (Fig. 2b), whereas the LPS-induced stabilization of the MyD88-downstream element p65 of the NFkB-pathway was not changed (Fig. 2c). Collectively, these findings suggest that flotillin-1 is involved in TLR3 but not in TLR4-mediated signaling.

The human flotillin family consists of two proteins with $46 \%$ amino acid homology, which form homo- and heterooligomers [2, 37]. Importantly, flotillin-2 shRNA had no effect on poly-I:C-induced adhesion molecule expression despite the fact that the shRNA drastically decreased the expression of flotillin-2 (Fig. 3a, b). These findings suggest that only flotillin-1 but not flotillin-2 is involved in TLR3 signaling.

Flotillin-1 is required for poly-I:C-induced adhesion of peripheral blood mononuclear cells on HUVEC

To determine the functional consequence of flotillin-1 depletion on poly-I:C signaling in HUVEC, adhesion assays were carried out with human peripheral blood mononuclear cells (PBMCs), mainly comprising lymphocytes and monocytes. The effect of flotillin shRNA on polymerase II by $2^{\wedge}-\Delta \Delta \mathrm{CT}$ in HUVEC stimulated with (each $10 \mu \mathrm{g} / \mathrm{ml}, 90 \mathrm{~min}$ ) or without LPS and poly-I:C after transduction with shRNAs for flotillin-1 (shFlot-1A and $B$ ), GFP (shGFP) or a scrambled shRNA (shScr). $N=3 ; * p<0.05$ with vs. without polyI.C, ${ }^{\#} p<0.05$ shGFP vs. shFlot

adhesion paralleled that on VCAM-1 expression: downregulation of flotillin-1, but not flotillin-2, drastically reduced PBMC adhesion on poly-I:C stimulated HUVEC (Fig. 3c, d).

Overexpression of flotillin-1 restores poly-I:C-induced VCAM-1 induction

As shRNA and siRNA in general may interfere with TLR3 signaling, experiments to test the specificity of our findings were carried out. Transfection of HUVECs with an shRNA-resistant flotillin-1-GFP expression plasmid restored normal poly-I:C-induced VCAM-1 induction (Fig. 3e). Interestingly, also in the absence of shRNA, overexpression of flotillin-1-GFP, but not flotillin-2-GFP promoted the poly-I:C-induced expression of VCAM-1 (Fig. 3f).

Flotillin-1 is involved in the uptake of poly-I:Crhodamine

For inflammatory cells, it has been suggested that TLRs differ in their cellular localization. Whereas TLR4-induced signaling occurs at the plasma membrane, TLR3, which is thought to mainly localize to endosomes, requires uptake of its ligands for signaling [23, 43]. Similarly, also in HUVECs, as determined by fluorescence-activated cell sorting 
A
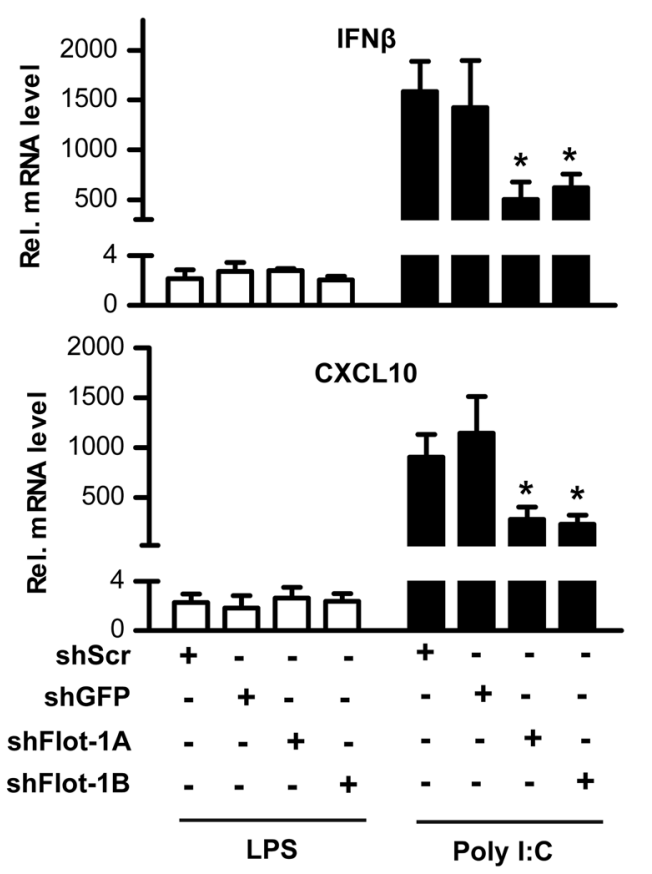

B
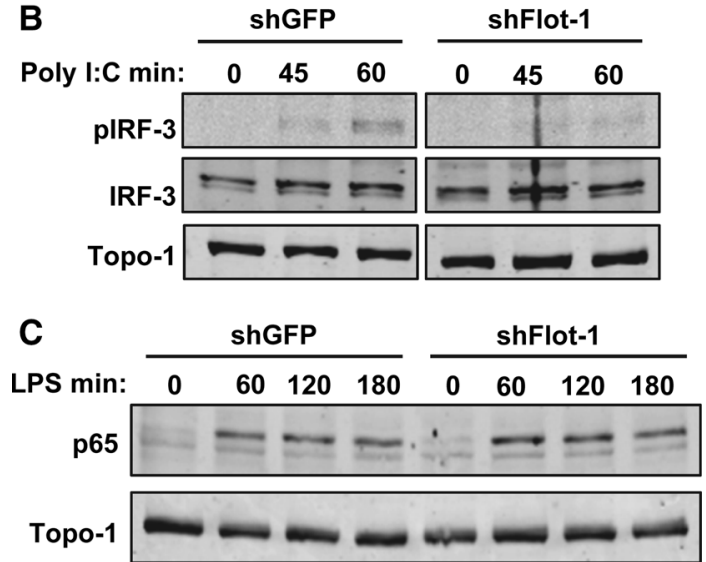

Fig. 2 Flotillin-1 is involved in poly-I:C-induced IRF-3 signaling. a qRT-PCR for the IRF-3-inducible genes IFN $\beta$ and CXCL10 in HUVEC stimulated with or without poly-I:C and LPS (each $10 \mu \mathrm{g} /$ $\mathrm{ml}, 90 \mathrm{~min}$ ) after transduction with shRNAs for flotillin-1 (shFlot$1 A$ and $B$ ), GFP (shGFP) or a scrambled shRNA (shScr). b and c Western blot analysis for the proteins indicated of nuclear extracts from HUVEC stimulated with poly-I:C (b, $10 \mu \mathrm{g} / \mathrm{ml})$ and LPS (c, $10 \mu \mathrm{g} / \mathrm{ml}$ ) for the times indicated after transduction with shRNA for flotillin-1 (shFlot-1) or GFP (shGFP). pIRF-3 indicated anti-phosphoIRF3 (phospho-Ser 385). Topo-1: DNA-topoisomerase. $N=4$; $* p<0.05$

(FACS), TLR4 was present on the surface of the cells whereas TLR3 was only detectable after permeabilization, indicative of an intracellular localization (Fig. S1). As flotillins are assumed to contribute to endocytosis and vesicular trafficking, it was determined whether flotillin-1 shRNA affects the transport of rhodamine-labeled polyI:C. In control cells, the poly-I:C-rhodamine fluorescence was readily detectable after $3 \mathrm{~h}$ stimulation, whereas in
shFlot-1 cells, poly-I:C uptake was greatly reduced (Fig. 4a, b). This suggests that flotillin-1 is involved in the trafficking of poly-I:C and thus in the delivery of dsRNA to its intracellular receptors.

The scavenger receptor SCARA4 mediates poly-I:Cinduced signaling in HUVEC

To facilitate endocytosis, uptake and delivery of poly-I:C to endosomes and thus to TLR3, cell surface receptors are required that bind the extracellular dsRNA. This task is carried out by scavenger receptors (SR). So far, eight structurally unrelated SR subclasses (A-H) [39] have been characterized of which subclasses A and B are thought to be the most relevant ones. We therefore screened the expression of SRs by qRT-PCR and found several of them to be highly expressed in HUVECs (Fig. 4c). To narrow down the selection to the ones relevant for poly-I:C binding, the effect of an SR A antagonist was tested. The SR A antagonizing polysaccharide polymer dextran sulfate [25] prevented the poly-I:C-induced VCAM-1 induction (Fig. 4d). This, in combination with the qRT-PCR screening data, make SCARA3 and SCARA4 likely candidates for poly-I:C binding. Subsequent siRNA experiments revealed that indeed the knockdown of SCARA4 but not SCARA3 prevented the poly-I:C-induced VCAM-1 induction (Fig. 4e). Thus, poly-I:C at the plasma membrane may bind to SCARA4, which could subsequently be internalized to transport poly-I:C to TLR3 in a process requiring flotillin-1.

\section{Flotillin-1 maintains structural proteins of caveolae}

To address the mechanism by which flotillin-1 interacts with the endocytotic pathways, proteomics was carried out. For this, the effect of flotillin shRNA on the protein composition of the detergent-resistant membrane protein fraction of HUVECs (Fig. S2a) was determined by stable isotope labeled amino acids (SILAC) in combination with ESI-Orbitrap mass spectrometry analysis. shRNA against the individual flotillins not only reduced their abundance in the detergent-resistant fraction, but also affected other proteins (Figs. 5a, S2b-d and Tables 1, S1-S3). Importantly, shRNA against flotillin-1 reduced the abundance of the structural caveolae proteins caveolin-1, cavin-1 and cavin-2 in the detergent-resistant fraction.

Flotillin-1 maintains caveolin-1 expression which is essential for the poly-I:C response

As caveolin-1, cavin-1 and cavin- 2 are essential for caveolae function $[16,18]$, it is plausible that the inhibitory effect of flotillin-1 shRNA on poly-I:C signaling is a 

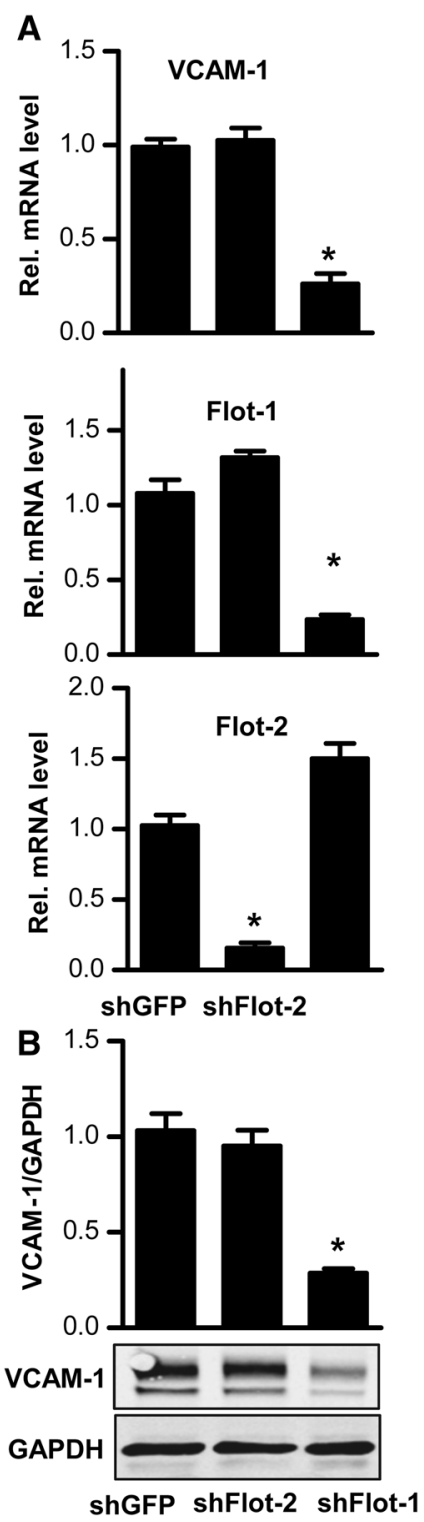

Fig. 3 Poly-I:C-induced adhesion of peripheral blood mononuclear cells requires flotillin-1. a qRT-PCR and b Western blot with densitometry for the gene indicated relative to the house-keeping gene RNA polymerase II or GAPDH in HUVECs stimulated with poly-I:C $(10 \mu \mathrm{g} / \mathrm{ml}$, a: $90 \mathrm{~min}, \mathbf{b}: 180 \mathrm{~min})$ after transduction with shRNA against flotillin-1 (shFlot-1), flotillin-2 (shFlot-2) or shGFP $N=4$, $p<0.05$. c and $\mathbf{d}$ Representative microscopic image (c) and statistical analysis (d) of peripheral blood mononuclear cell (PBMCs) adhering

consequence of caveolae dysfunction. Indeed, siRNA against caveolin-1, similar to flotillin-1 shRNA, attenuated caveolin-1 expression which was accompanied by decreased poly-I:C-induced VCAM-1 expression (Fig. 5b). It appears that flotillin-1 maintains caveolin-1 level, as also in whole cell lysates, flotillin-1 shRNA but not flotillin-2 depletion decreased caveolin-1 protein levels in HUVECs and in mouse lung endothelial cells (Figs. 5b, S3a, b). Also, overexpression of caveolin-1 in shFlot-1 cells failed
D

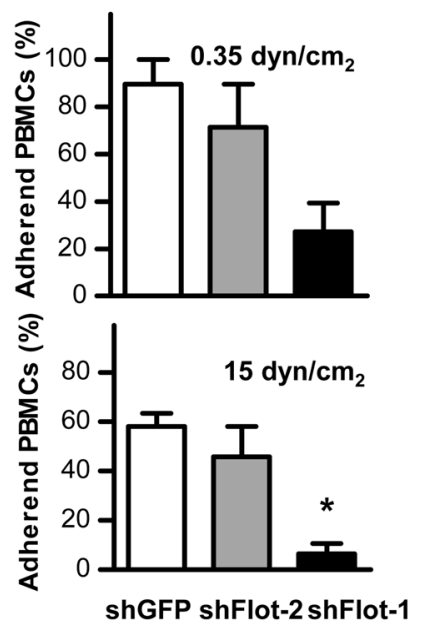




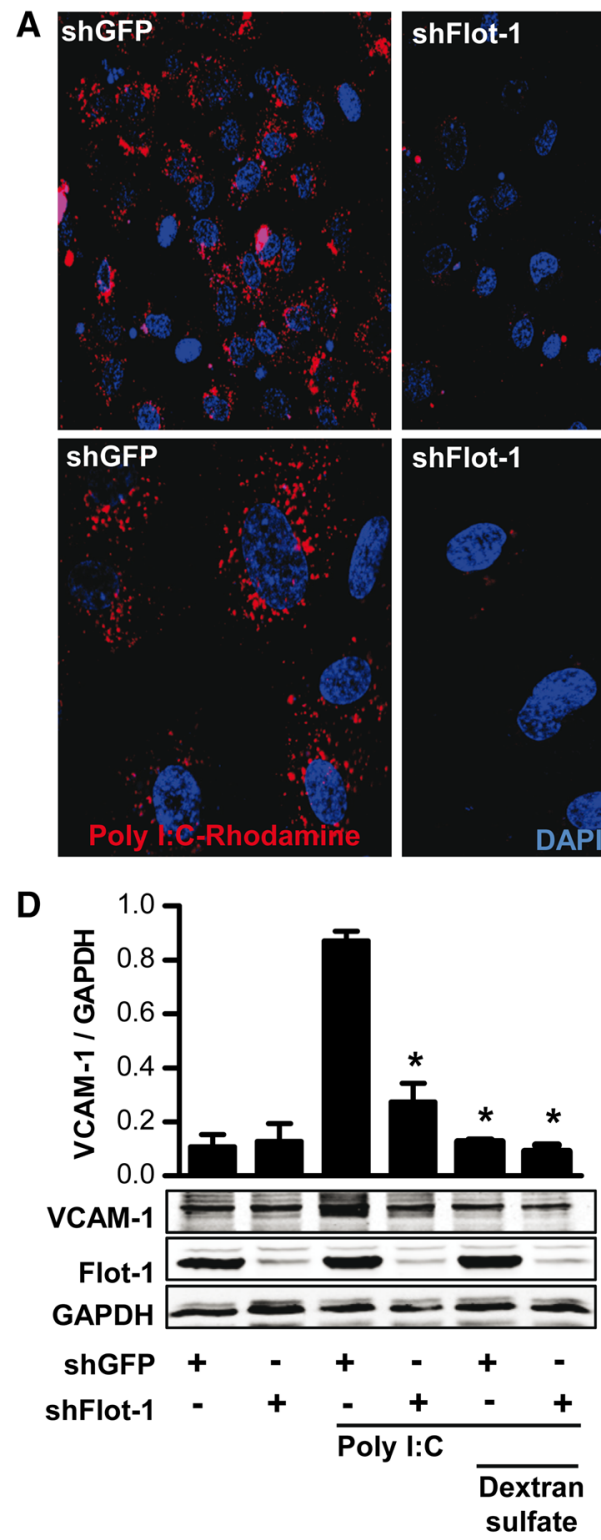

B

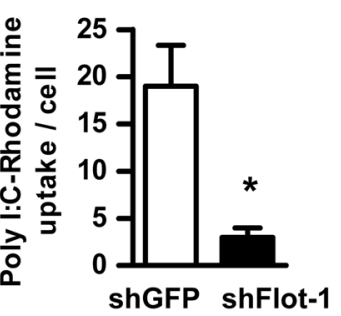

$\mathbf{E}$
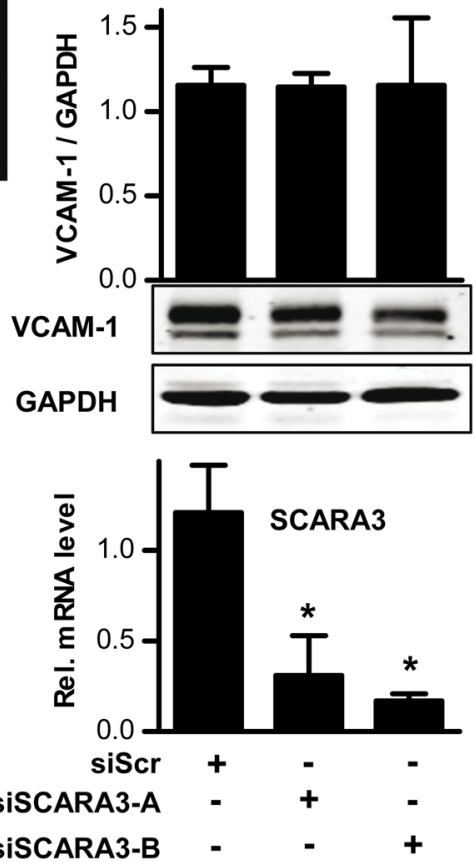

C

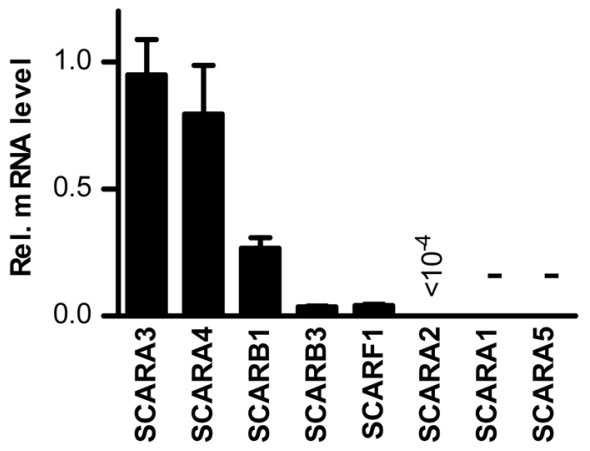

Fig. 4 The scavenger receptor SCARA4 mediates poly-I:C-induced signaling. $\mathbf{a}$ and $\mathbf{b}$ Original laser scanning microscopic images (a) and quantification (b) from HUVEC transduced with shGFP and shFlot-1 after incubation with poly-I:C-rhodamine ( $r e d, 0.5 \mu \mathrm{g} / \mathrm{ml}, 180 \mathrm{~min}$ ). Nuclei are counterstained with DAPI (blue). c q-RT-PCR for the genes indicated relative to the house-keeping gene RNA polymerase II in HUVECs. d Representative Western blot and densitometry for the genes indicated in HUVECs transduced with shGFP or shFlot-1 after pretreatment with and without the class A scavenger receptor

flotillin-1 interferes with caveolin-1 stability. However, the inhibitory effect of flotillin-1 shRNA on caveolin-1 was not prevented by inhibition of the proteasome or the lysosomal pathway (Fig. S3d, e). Also, flotillin-1 shRNA had only a small, yet significant, effect on caveolin-1 mRNA expression (Fig. S3f) suggesting rather attenuated translation and/ or misfolding caveolin-1 protein. Disturbing of endoplasmic reticulum (ER) protein formation and maturation leads inhibitor dextran sulfate $(10 \mu \mathrm{g} / \mathrm{ml}, 5 \mathrm{~min})$ and subsequent stimulation with poly-I:C $(10 \mu \mathrm{g} / \mathrm{ml}, 180 \mathrm{~min}) . N=4,{ }^{*} p<0.05$. e Western blot analysis and densitometry for the proteins indicated and qRTPCR for SCARA3 (left bottom) and SCARA4 (right bottom) in HUVECs treated with scrambled siRNA or two different siRNAs ( - A and -B) against SCARA3 (left, siSCARA3) or SCARA4 (right, siSCARA4) followed by stimulation with poly-I:C $(10 \mu \mathrm{g} / \mathrm{ml}, 180)$. $N=4, * p<0.05$

to the ER stress also termed unfolded protein response (URP), a situation in which ER-localized translation is attenuated. ER stress activates a signaling pathway which includes phosphorylation of the type I ER transmembrane kinase PERK which then directly phosphorylates the eukaryotic initiation factor 2 eIF $2 \alpha$. Importantly, increased PERK and eIF2 $\alpha$ phosphorylation were present in shFlot-1 cells (Fig. 5d), indicating that loss of flotillin-1 results in 


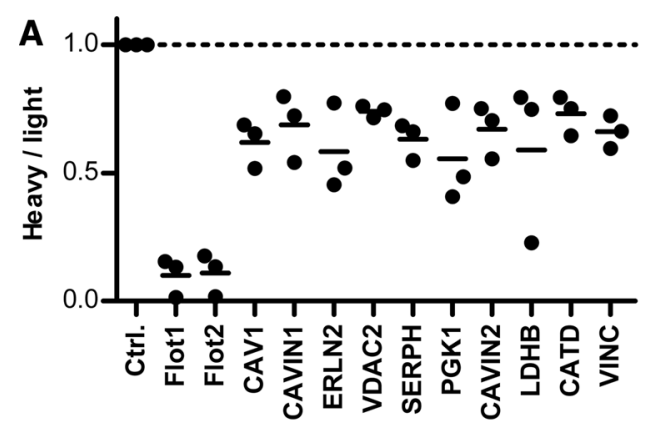

B
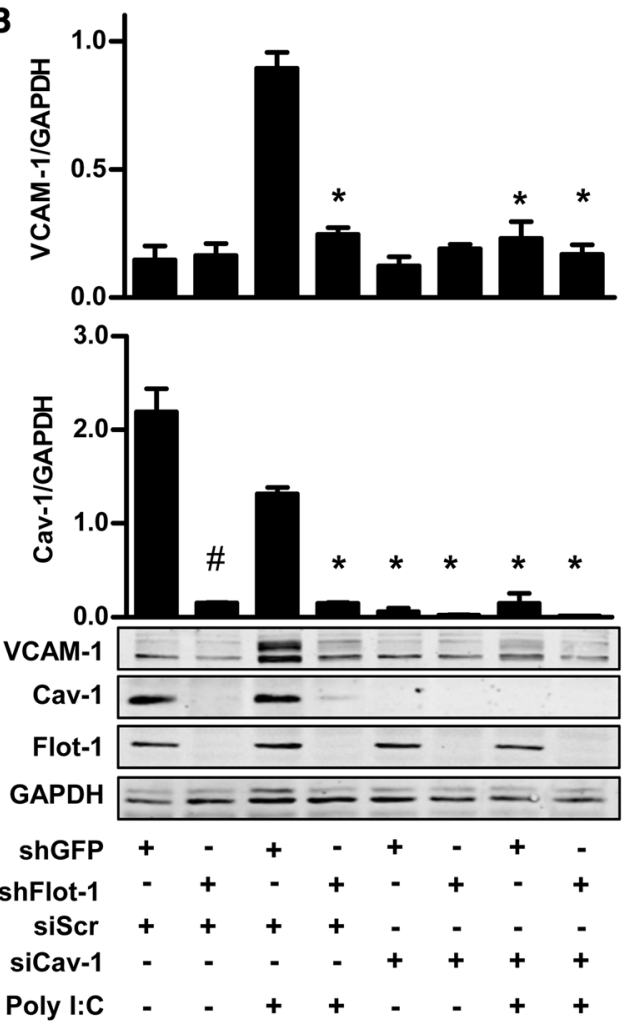

$\mathbf{E}$

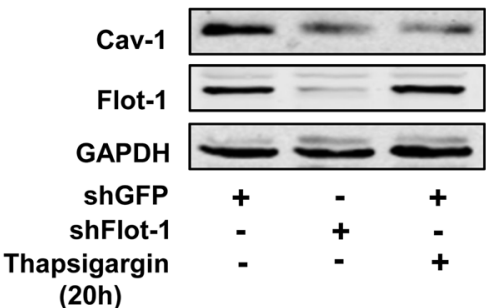

Fig. 5 Flotillin-1 maintains caveolin-1 protein which is required for the poly-I:C response. a Subset of SILAC-mass spectrometric analyses on the effect of shFlot-1 on protein abundance in the detergent-resistant membrane fraction of HUVECs. b Exemplary Western blot and densitometry for the proteins indicated of HUVECs transduced with shGFP or shFlot-1 or transfects with scrambled siRNA (siScr) or caveolin-1 siRNA (siCav-1) with and without stimulation with poly-I:C $(10 \mu \mathrm{g} / \mathrm{ml}, 180 \mathrm{~min}),{ }^{*} p<0.05, n=4$.

D
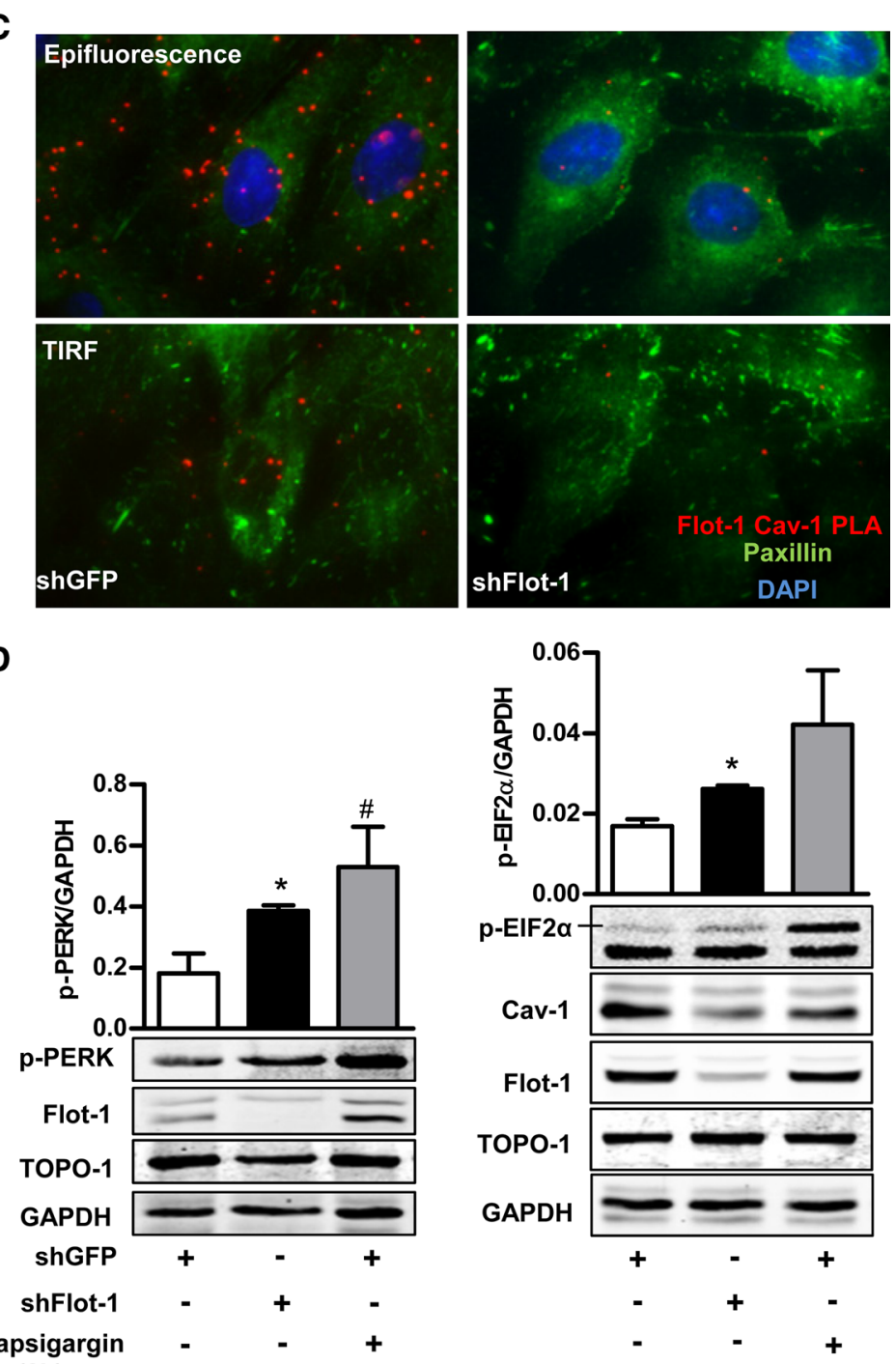

(2h)

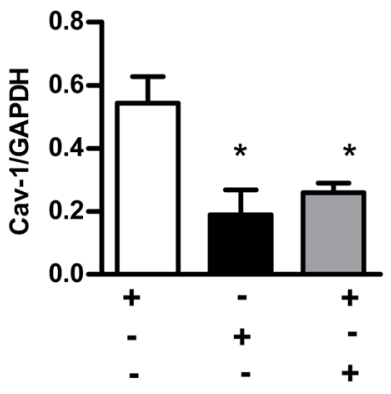

c Proximity ligation assays for flotillin-1 and caveolin-1 interaction (red) in HUVECs transduced with shGFP or shFlotillin-1. Down total internal reflection fluorescence microscopy (TIRF) image, up epifluorescence. Cells were counterstained with paxillin (green) and DAPI (blue). d and e Exemplary Western blot and densitometry for the proteins indicated of HUVECs transduced with shGFP or shFlot-1 with and without stimulation of thapsigargin $3.3 \mu \mathrm{mol} / 1 . \quad N=4$ $* p<0.05$ 
Table 1 Flotillin-1 SILAC LTQ-Orbitrap-MS: list of proteins significantly down-regulated $(>20 \%)$ by shFlot- 1 relative to shGFP in the detergent-resistant membrane of HUVECs $(n=3)$

\begin{tabular}{|c|c|c|c|c|c|}
\hline Protein & & Accession & Score & $\begin{array}{l}\text { Mean } \\
\text { heavy/ } \\
\text { light }\end{array}$ & SEM \\
\hline Flot1 & Flotillin-1 & O75955 & 1,934 & 0.10 & 0.04 \\
\hline Flot2 & Flotillin-2 & Q14254 & 1,794 & 0.11 & 0.05 \\
\hline CAV1 & Caveolin-1 & Q03135 & 1,362 & 0.62 & 0.05 \\
\hline CAVIN1 & Cavin-1 & Q6NZI2 & 1,111 & 0.69 & 0.08 \\
\hline ERLN2 & Erlin-2 & O94905 & 793 & 0.58 & 0.10 \\
\hline VDAC2 & $\begin{array}{l}\text { Isoform } 2 \text { of } \\
\text { voltage-dependent } \\
\text { anion-selective } \\
\text { channel protein } 2\end{array}$ & P45880-2 & 623 & 0.74 & 0.01 \\
\hline SERPH & Serpin H1 & P50454 & 512 & 0.63 & 0.04 \\
\hline PGK1 & $\begin{array}{l}\text { Phosphoglycerate } \\
\text { kinase } 1\end{array}$ & P00558 & 419 & 0.55 & 0.11 \\
\hline CAVIN2 & Cavin-2 & O95810 & 418 & 0.67 & 0.06 \\
\hline LDHB & $\begin{array}{l}\text { L-lactate } \\
\text { dehydrogenase B } \\
\text { chain }\end{array}$ & P07195 & 333 & 0.59 & 0.18 \\
\hline CATD & Cathepsin D & P07339 & 268 & 0.73 & 0.04 \\
\hline VINC & Vinculin & P18206-2 & 167 & 0.66 & 0.04 \\
\hline
\end{tabular}

ER stress, which then may block caveolin-1 translation. Accordingly, treatment of HUVEC with the ER-stress inducer thapsigargin similarly abolished caveolin-1 expression independent of the expressions level of flotillin1 (Fig. 5e). In keeping with this, after down-regulation of flotillin-1 even plasmid-based overexpression of caveolin-1 failed to restore the normal protein levels (Fig. S3c).

To substantiate our hypothesis that caveolae are required for poly-I:C signaling, cholesterol-perturbing agents were studied. Although this approach is unspecific, interfering with caveolae function by methyl-beta-cyclodextrin, filipin and carrageenan attenuated the poly-I:C-induced VCAM-1 expression (Fig. S4). Collectively, flotillin-1 is required for caveolin-1 expression in endothelial cells, and lack of caveolin-1 upon down-regulation of flotillin-1 results in an inhibition of caveolae function which is required for polyI:C signaling.

\section{Discussion}

In this study, we determined the contribution of flotillin-1 to endothelial inflammatory TLR signaling. Flotillin-1 was required for poly-I:C-dependent signaling through TLR3 but not for LPS-mediated signaling through TLR4. Flotillin-1, but not flotillin-2 facilitated TLR3-mediated IRF activation and the subsequent expression of adhesion molecules which leads to leukocyte attachment. As potential mechanism we identified that flotillin-1 maintains the cellular caveolin-1 protein content.

The exact mechanisms by which extracellular dsRNA and poly-I:C are bound on the cell surface and gain entry into the cell are poorly characterized. Scavenger receptors of class A and B are likely candidates due to their cell surface expression and ability to bind nucleic acids $[9,11]$. Our screen revealed that both scavenger receptor classes are expressed in HUVECs and particularly SCARA4 appeared to be important for poly-I:C-mediated signaling. Thus, it can be inferred that SCARA4 acts as carrier for poly-I:C and delivers the molecule to TLR3. The process presumably requires flotillin-1, as the uptake of poly-I:C was reduced after flotillin-1 down-regulation. Our data suggest, however, that flotillin-1 acts only indirectly by maintaining caveolin-1, which forms the structural correlate of the endocytotic pathway of relevance here.

Caveolae are highly abundant in the endothelium and make up to $20 \%$ of the surface of the cell [16]. One of their major functions is the clathrin-independent endocytosis, a process which requires caveolin proteins [18]. Caveolin-1 acts as a scaffolding protein that facilitates caveolae formation and endocytosis [22]. In endothelial cells, caveolin-1 has complex roles in signaling due to its multiple functions. Translocation of enzymes like endothelial NO synthase into caveolae reduced their activity [34], whereas translocation of receptors and kinases into caveolae can lead to the formation of signaling hot spots [42]. Finally, caveolae-mediated uptake of receptors results in termination of signaling or facilitates intracellular signaling [3].

Our data demonstrate that in the case of poly-I:Cinduced signaling, the latter is true: Cholesterol perturbing reagents which induce caveolae dysfunction blocked the poly-I:C-mediated induction of adhesion molecules, and caveolin-1 siRNA had a similar effect. These findings mimic the effect of down-regulation of flotillin-1. The reason for this was that flotillin-1 maintained caveolin-1, cavin-1 and cavin-2 in the detergent-resistant membrane fraction, generally considered containing caveolae and other membrane rafts. It should be emphasized that we did not intend to directly characterize caveolae or lipid rafts by the proteomics approach in the present study but rather sought for the effect of depletion of flotillins on the detergent-resistant membrane fraction. We chose this approach as detergent-free isolation of lipid rafts and caveolae in our hands were too variable for proteomics which is naturally limited in the number of samples applicable and in sensitivity. Thus, although we could clearly identify that flotillin-1 impacts on caveolar proteins, we cannot make statements concerning the consequence on caveolae at the plasma membrane itself. Subsequent Western blots revealed that flotillin-1 knock-down resulted 
in a global depletion of caveolin-1 from the cell, which may suggest that already at an early stage of membrane integration of caveolin-1, flotillin-1 might be of relevance. The combination of proximity ligation assay and TIRF technique in our study supports this view: although flotillins-1 co-localized with caveolin-1 to some extent, this effect was largely restricted to intracellular compartments around the nucleus, but not frequent at the plasma membrane. Our finding of a very restricted intracellular interaction of flotillin-1 and caveolin-1 may also help to explain why by means of membrane isolation or conventional LSM technique, interactions of the two proteins were not reported $[12,13]$. The fact that the proteins can be coimmuno-precipitated [41] does not contradict this notion as it is probably not possible to successfully solubilize membrane rafts without disrupting antibody-antigen binding. Thus, in immunoprecipitation studies usually whole membrane patches are being pulled down.

In the present study, we found no evidence that flotillin-1 prevents the degradation of caveolin-1 as neither lysosomal nor proteasomal inhibitors restored caveolin-1 level in flotillin-1 knock-down cells. Although this contrasts a previous observation in an epithelial cell line [40] it points to a function of flotillins-1 in caveolin-1 synthesis. Depletion of flotillin-1 in HUVEC induced a small decrease in caveolin1 mRNA expression which, however, is not sufficient to explain the almost complete lack of caveolin-1 protein under this condition. Therefore, we assumed that the lack of flotillins-1 prevents de novo synthesis of caveolin-1 at the level of translation. The homeostasis of the endoplasmic reticulum (ER) is important for maturation of newly synthesized secretory and transmembrane proteins. Conditions interfering with the function of ER are called ER stress. ER stress is induced by accumulation of unfolded proteins (unfolded protein response, UPR) or by excessive protein demand caused by viral infection (ER overload response, EOR) $[19,21]$. Activation of ER stress sensors such as PERK kinase or EIF2 $\alpha$ reduces the ER workload and induces expression of chaperons. Our findings that flotillin1 depletion induces ER stress signaling and that caveolin-1 translation itself is sensitive to ER stress explain its reduced level in shFlot-1 HUVECs.

The question remains how flotillin-1 depletion induces ER-stress. Flotillin-1 is involved in endosomal sorting, trafficking from and towards the plasma membrane and the transport to the ER and Golgi $[29,35]$. We speculate that the lack of flotillin-1 disturbs vesicular trafficking but further studies will be needed to clarify this aspect.

Flotillin-2 can stabilize flotillin-1 $[2,13,15]$ and also in HUVEC, flotillin- 2 shRNA reduced the flotillin-1 protein expression to some extent. Despite this, loss of flotillin-2 often does not phenocopy the loss of flotillins-1 [7]. For example, ERK signaling is increased in flotillin-2 knockout mice whereas depletion of flotillin-1 decreased it [5]. Also in the present study, depletion of flotillin-2 reduced flotillin-1 expression to some extent but was without effect on signaling. Potentially, the residual flotillin-1 expression was sufficient to maintain signaling. Our flotillin-1 overexpression experiments at least exclude that the antiinflammatory effect of flotillin-1 shRNA was mediated by excess flotillin-2 exhibiting aberrant binding.

It is a limitation of the present study that only cultured cells were studied but not knockout mice. In fact, such experiments were carried out by us, but in keeping with the overall very mild phenotype in the flotillin- 1 global knockout mice [7, 26], we failed to detect a difference in caveolin1 expression between wild type and flotillin-1 global knockouts (C. Fork. unpublished observations). Thus, it was important to exclude that the altered responses in the cells were not a consequence of an unspecific interference of the shRNAs and siRNAs with TLR signaling. The fundamental experiments were performed in two different shRNAs with similar results and overexpression of flotillin-1 restored normal poly-I:C-induced VCAM-1 expression. To exclude that the lack of effect in the global knockout mice was a consequence of a specific function of flotillin-1 in HUVEC, the key experiments, i.e. the attenuation of poly-I:C induced VCAM-1 induction by flotillin-1 shRNA, was also carried out in murine lung endothelial cells, with similar results. We therefore conclude that so far unidentified compensations beyond the scope of our present study are operative in global flotillin-1 knockout mouse.

\section{Conclusion}

We have identified that flotillin-1 is an important mediator of TLR3 signaling in HUVEC. In these cells, acute loss of flotillin-1 leads to attenuation of caveolar protein abundance. As caveolar proteins turn out to be required for TLR3 signaling, flotillin-1 might represent a novel target for anti-inflammatory therapy.

Acknowledgment The authors are grateful for the excellent technical support by Susanne Schütz, Cindy Höper, Tanja Lüneburg and Katalin Palfi. This work was funded by the Faculty of Medicine of Goethe-University, Frankfurt am Main and the DFG excellence cluster (EX-147) Cardio-Pulmonary System (ECCPS), and the German Center for Cardiovascular Research (DZHK).

Conflict of interest On behalf of all authors, the corresponding author states that there is no conflict of interest.

\section{References}

1. Amaddii M, Meister M, Banning A, Tomasovic A, Mooz J, Rajalingam K, Tikkanen R (2012) Flotillin-1/reggie-2 protein 
plays dual role in activation of receptor-tyrosine kinase/mitogenactivated protein kinase signaling. J Biol Chem 287:7265-7278. doi:10.1074/jbc.M111.287599

2. Babuke T, Ruonala M, Meister M, Amaddii M, Genzler C, Esposito A, Tikkanen R (2009) Hetero-oligomerization of reggie-1/ flotillin-2 and reggie-2/flotillin-1 is required for their endocytosis. Cell Signal 21:1287-1297. doi:10.1016/j.cellsig.2009.03.012

3. Balogh P, Katz S, Kiss AL (2013) The role of endocytic pathways in TGF-beta signaling. Pathol Oncol Res 19:141-148. doi:10. 1007/s12253-012-9595-8

4. Banning A, Ockenga W, Finger F, Siebrasse P, Tikkanen R (2012) Transcriptional regulation of flotillins by the extracellularly regulated kinases and retinoid X receptor complexes. PLoS One 7:e45514. doi:10.1371/journal.pone.0045514

5. Banning A, Regenbrecht CR, Tikkanen R (2014) Increased activity of mitogen activated protein kinase pathway in flotillin-2 knockout mouse model. Cell Signal 26:198-207. doi:10.1016/j. cellsig.2013.11.001

6. Baumann CA, Ribon V, Kanzaki M, Thurmond DC, Mora S, Shigematsu S, Bickel PE, Pessin JE, Saltiel AR (2000) CAP defines a second signalling pathway required for insulin-stimulated glucose transport. Nature 407:202-207. doi:10.1038/ 35025089

7. Bitsikas V, Riento K, Howe JD, Barry NP, Nichols BJ (2014) The role of flotillins in regulating abeta production, investigated using flotillin 1-/-, flotillin 2-/- double knockout mice. PLoS One 9:e85217. doi:10.1371/journal.pone.0085217

8. Carcea I, Ma'ayan A, Mesias R, Sepulveda B, Salton SR, Benson DL (2010) Flotillin-mediated endocytic events dictate cell typespecific responses to semaphorin 3A. J Neurosci 30:15317-15329. doi:10.1523/JNEUROSCI.1821-10.2010

9. Dieudonne A, Torres D, Blanchard S, Taront S, Jeannin P, Delneste Y, Pichavant M, Trottein F, Gosset P (2012) Scavenger receptors in human airway epithelial cells: role in response to double-stranded RNA. PLoS One 7:e41952. doi:10.1371/journal. pone. 0041952

10. El-Sayed A, Harashima H (2013) Endocytosis of gene delivery vectors: from clathrin-dependent to lipid raft-mediated endocytosis. Mol Ther 21:1118-1130. doi:10.1038/mt.2013.54

11. Ezzat K, Helmfors H, Tudoran O, Juks C, Lindberg S, Padari K, El-Andaloussi S, Pooga M, Langel U (2012) Scavenger receptormediated uptake of cell-penetrating peptide nanocomplexes with oligonucleotides. FASEB J 26:1172-1180. doi:10.1096/fj.11191536

12. Fernow I, Icking A, Tikkanen R (2007) Reggie-1 and reggie-2 localize in non-caveolar rafts in epithelial cells: cellular localization is not dependent on the expression of caveolin proteins. Eur J Cell Biol 86:345-352. doi:10.1016/j.ejcb.2007.03.004

13. Frick M, Bright NA, Riento K, Bray A, Merrified C, Nichols BJ (2007) Coassembly of flotillins induces formation of membrane microdomains, membrane curvature, and vesicle budding. Curr Biol 17:1151-1156. doi:10.1016/j.cub.2007.05.078

14. Ge L, Qi W, Wang LJ, Miao HH, Qu YX, Li BL, Song BL (2011) Flotillins play an essential role in Niemann-Pick C1-like 1-mediated cholesterol uptake. Proc Natl Acad Sci USA 108:551-556. doi:10.1073/pnas.1014434108

15. Glebov OO, Bright NA, Nichols BJ (2006) Flotillin-1 defines a clathrin-independent endocytic pathway in mammalian cells. Nat Cell Biol 8:46-54. doi:10.1038/ncb1342

16. Hansen CG, Shvets E, Howard G, Riento K, Nichols BJ (2013) Deletion of cavin genes reveals tissue-specific mechanisms for morphogenesis of endothelial caveolae. Nat Commun 4:1831. doi:10.1038/ncomms 2808

17. Kim KB, Lee JS, Ko YG (2008) The isolation of detergentresistant lipid rafts for two-dimensional electrophoresis. Methods Mol Biol 424:413-422. doi:10.1007/978-1-60327-064-9_32
18. Kirkham M, Nixon SJ, Howes MT, Abi-Rached L, Wakeham DE, Hanzal-Bayer M, Ferguson C, Hill MM, Fernandez-Rojo M, Brown DA, Hancock JF, Brodsky FM, Parton RG (2008) Evolutionary analysis and molecular dissection of caveola biogenesis. J Cell Sci 121:2075-2086. doi:10.1242/jcs.024588

19. Kuang E, Wan Q, Li X, Xu H, Liu Q, Qi Y (2005) ER $\mathrm{Ca} 2+$ depletion triggers apoptotic signals for endoplasmic reticulum (ER) overload response induced by overexpressed reticulon 3 (RTN3/HAP). J Cell Physiol 204:549-559. doi:10. $1002 /$ jcp. 20340

20. Lafyatis R, York M, Marshak-Rothstein A (2006) Antimalarial agents: closing the gate on toll-like receptors? Arthritis Rheum 54:3068-3070. doi:10.1002/art.22157

21. Lawless MW, Greene CM, Mulgrew A, Taggart CC, O’Neill SJ, McElvaney NG (2004) Activation of endoplasmic reticulumspecific stress responses associated with the conformational disease $\mathrm{Z}$ alpha 1-antitrypsin deficiency. J Immunol 172:5722-5726

22. Le LS, Kurzchalia TV (2005) Getting rid of caveolins: phenotypes of caveolin-deficient animals. Biochim Biophys Acta 1746:322-333. doi:10.1016/j.bbamcr.2005.06.001

23. Lee MS, Kim YJ (2007) Signaling pathways downstream of pattern-recognition receptors and their cross talk. Annu Rev Biochem 76:447-480. doi:10.1146/annurev.biochem.76.060605. 122847

24. Lee TH, McKleroy W, Khalifeh-Soltani A, Sakuma S, Lazarev S, Riento K, Nishimura SL, Nichols BJ, Atabai K (2014) Functional genomic screen identifies novel mediators of collagen uptake. Mol Biol Cell 25:583-593. doi:10.1091/mbc.E13-07-0382

25. Limmon GV, Arredouani M, McCann KL, Corn Minor RA, Kobzik L, Imani F (2008) Scavenger receptor class-A is a novel cell surface receptor for double-stranded RNA. FASEB J 22:159-167. doi:10.1096/fj.07-8348com

26. Ludwig A, Otto GP, Riento K, Hams E, Fallon PG, Nichols BJ (2010) Flotillin microdomains interact with the cortical cytoskeleton to control uropod formation and neutrophil recruitment. J Cell Biol 191:771-781. doi:10.1083/jcb.201005140

27. Lundberg AM, Drexler SK, Monaco C, Williams LM, Sacre SM, Feldmann M, Foxwell BM (2007) Key differences in TLR3/poly I: C signaling and cytokine induction by human primary cells: a phenomenon absent from murine cell systems. Blood 110:3245-3252. doi:10.1182/blood-2007-02-072934

28. McGettrick AF, O'Neill LA (2010) Localisation and trafficking of toll-like receptors: an important mode of regulation. Curr Opin Immunol 22:20-27. doi:10.1016/j.coi.2009.12.002

29. Meister M, Tikkanen R (2014) Endocytic trafficking of membrane-bound cargo: a flotillin point of view. Membranes (Basel) 4:356-371. doi:10.3390/membranes4030356

30. Neumann-Giesen C, Fernow I, Amaddii M, Tikkanen R (2007) Role of EGF-induced tyrosine phosphorylation of reggie-1/flotillin-2 in cell spreading and signaling to the actin cytoskeleton. J Cell Sci 120:395-406. doi:10.1242/jcs.03336

31. Ospelt C, Gay S (2010) TLRs and chronic inflammation. Int J Biochem Cell Biol 42:495-505. doi:10.1016/j.biocel.2009.10.010

32. Otto GP, Nichols BJ (2011) The roles of flotillin microdomainsendocytosis and beyond. J Cell Sci 124:3933-3940. doi:10.1242/ jes.092015

33. Pust S, Dyve AB, Torgersen ML, van DB, Sandvig K (2010) Interplay between toxin transport and flotillin localization. PLoS One 5:e8844. doi:10.1371/journal.pone.0008844

34. Ramadoss J, Pastore MB, Magness RR (2013) Endothelial caveolar subcellular domain regulation of endothelial nitric oxide synthase. Clin Exp Pharmacol Physiol 40:753-764. doi:10.1111/ 1440-1681.12136

35. Saslowsky DE, Cho JA, Chinnapen H, Massol RH, Chinnapen DJ, Wagner JS, De Luca HE, Kam W, Paw BH, Lencer WI (2010) Intoxication of zebrafish and mammalian cells by cholera 
toxin depends on the flotillin/reggie proteins but not Derlin-1 or -2. J Clin Invest 120:4399-4409. doi:10.1172/JCI42958

36. Schneider A, Rajendran L, Honsho M, Gralle M, Donnert G, Wouters F, Hell SW, Simons M (2008) Flotillin-dependent clustering of the amyloid precursor protein regulates its endocytosis and amyloidogenic processing in neurons. J Neurosci 28:2874-2882. doi:10.1523/JNEUROSCI.5345-07.2008

37. Solis GP, Hoegg M, Munderloh C, Schrock Y, Malaga-Trillo E, Rivera-Milla E, Stuermer CA (2007) Reggie/flotillin proteins are organized into stable tetramers in membrane microdomains. Biochem J 403:313-322. doi:10.1042/BJ20061686

38. Solis GP, Hulsbusch N, Radon Y, Katanaev VL, Plattner H, Stuermer CA (2013) Reggies/flotillins interact with Rab11a and SNX4 at the tubulovesicular recycling compartment and function in transferrin receptor and E-cadherin trafficking. Mol Biol Cell 24:2689-2702. doi:10.1091/mbc.E12-12-0854

39. Sorensen KK, McCourt P, Berg T, Crossley C, Le CD, Wake K, Smedsrod B (2012) The scavenger endothelial cell: a new player in homeostasis and immunity. Am J Physiol Regul Integr Comp Physiol 303:R1217-R1230. doi:10.1152/ajpregu.00686.2011

40. Vassilieva EV, Ivanov AI, Nusrat A (2009) Flotillin-1 stabilizes caveolin-1 in intestinal epithelial cells. Biochem Biophys Res Commun 379:460-465. doi:10.1016/j.bbrc.2008.12.118

41. Volonte D, Galbiati F, Li S, Nishiyama K, Okamoto T, Lisanti MP (1999) Flotillins/cavatellins are differentially expressed in cells and tissues and form a hetero-oligomeric complex with caveolins in vivo. Characterization and epitope-mapping of a novel flotillin-1 monoclonal antibody probe. J Biol Chem 274:12702-12709

42. Williams JJ, Palmer TM (2014) Cavin-1: caveolae-dependent signalling and cardiovascular disease. Biochem Soc Trans 42:284-288. doi:10.1042/BST20130270

43. Yamashita M, Chattopadhyay S, Fensterl V, Zhang Y, Sen GC (2012) A TRIF-independent branch of TLR3 signaling. J Immunol 188:2825-2833. doi:10.4049/jimmunol.1103220 